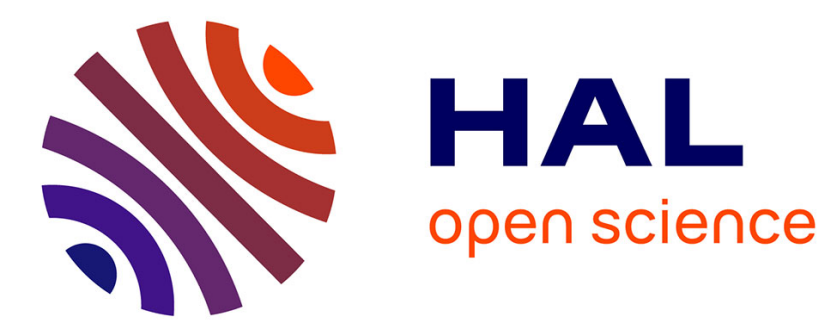

\title{
Molecular dynamics simulation of ionic conductors: perspectives and limitations
}

\author{
Dirk Zahn
}

\section{To cite this version:}

Dirk Zahn. Molecular dynamics simulation of ionic conductors: perspectives and limitations. Journal of Molecular Modeling, 2010, 17 (7), pp.1531-1535. 10.1007/s00894-010-0877-3 . hal-00637163

\section{HAL Id: hal-00637163 https://hal.science/hal-00637163}

Submitted on 31 Oct 2011

HAL is a multi-disciplinary open access archive for the deposit and dissemination of scientific research documents, whether they are published or not. The documents may come from teaching and research institutions in France or abroad, or from public or private research centers.
L'archive ouverte pluridisciplinaire HAL, est destinée au dépôt et à la diffusion de documents scientifiques de niveau recherche, publiés ou non, émanant des établissements d'enseignement et de recherche français ou étrangers, des laboratoires publics ou privés. 


\title{
Molecular dynamics simulation of ionic conductors: perspectives and limitations
}

Received: 20.09.2010 / Accepted: 30.09.2010

Dirk Zahn

Lehrstuhl für Theoretische Chemie / Computer Chemie Centrum, Universität ErlangenNürnberg, Nägelsbachstr. 25, 91052 Erlangen, Germany

Email: dirk.zahn@chemie.uni-erlangen.de

\begin{abstract}
Concepts of modeling ion conductors by means of molecular dynamics simulations are outlined and exemplified by a small series of applications. Computational studies offer atomic level of detail insights into ionic mobility and thus help to rationalize experimental findings in particular for complex ion conduction mechanisms. Standard techniques are discussed within their thermodynamic context i.e. chemical potentials, conductivity and activation energies. The limitations of molecular dynamics simulations are defined by statistics and the accuracy in evaluating atomic interactions.
\end{abstract}

Keywords Ionic conductors $\cdot$ Molecular dynamics 


\section{Introduction}

The phenomenon of ion conductivity in solids is of tremendous technical importance. To support the experimentalists search for compounds of high conductivity and their characterization, atomistic simulation models are becoming increasingly popular. The profound understanding of transport phenomena in ionic devices requires in-situ investigations at the atomistic level of detail. While hard to achieve from experiment, molecular dynamics (MD) simulations may provide such a tool of investigation.

So far, MD simulations related to ion conductivity were mainly focused on either singlecrystalline or amorphous systems. The accessible insights include the mechanisms of Frenkel defect formation, interstitial and/or vacancy migration and recombination. Inspired by experimental work on nanoscale ionic conductors [1-3], recent MD simulations also addressed nanoionics systems [4-6]. For such systems, also structural insights may be derived from simulation [4].

While MD simulations offer understanding at atomic level of detail and the calculation of experimentally assessable properties such as conductivity, chemical potentials and activation energies, there is a number of technical complications that still limit the elsewise quite impressive potential of MD simulations. Here, we outline standard and more specific techniques to model ion conductors.

\section{Molecular dynamics simulations}

Based on ab initio or empirically derived forces acting between the atoms, MD simulations allow the time propagation of atomic model systems [7]. As the latter are typically large, numerical approaches to solve the Newton's equations of motions are employed. The key idea is to divide a trajectory into many small time intervals. Within such an interval, the forces are assumed to be constant, hence allowing the prediction of atomic positions $r$ at time $t+\Delta t$ on the basis of positions, velocities and acceleration at time $t$. The Verlet algorithm reads [8]: 


$$
\begin{aligned}
& \frac{d^{2} r_{i}}{d t^{2}}=\frac{r_{i}\left(+\Delta t>2 r_{i}\left(\exists r_{i}(-\Delta t)\right.\right.}{\Delta t^{2}}+O\left(\Delta t^{4}\right)
\end{aligned}
$$

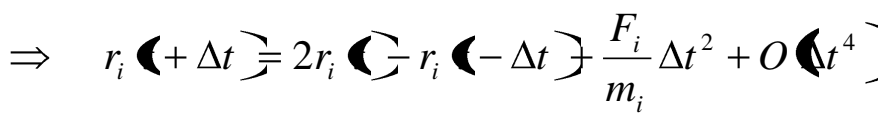

where the index $i$ runs over all atoms of the model system. To keep the error $\mathrm{O}\left(\Delta \mathrm{t}^{4}\right)$ negligible, $\Delta \mathrm{t}$ has to be chosen rather small, typically around $1 \mathrm{fs}$. Longer trajectories are produced from using Eq. 1 iteratively, hence positions, velocities and forces are calculated in time intervals using small time steps of $\Delta \mathrm{t}=0.1-5 \mathrm{fs}$, depending on atomic masses and the applied temperature.

To mimic realistic simulation conditions, MD simulations may be coupled to algorithms that scale the simulation cell and the atomic velocities to induce specific values of pressure and temperature, respectively [7].

\section{Modeling of atomic interactions}

The atomic interaction forces may be derived from quantum mechanical calculations or from less accurate, but less expensive empirical interaction models (force fields). While quantum calculations are accurate in evaluating the forces acting on a specific configuration, this does not imply that $a b$ initio MD simulations would automatically provide the most reliable results. Indeed, with the currently accessible hardware many properties may be predicted more accurately when using empirical force-fields. This apparent contradiction is due to statistical errors. The large computational costs related to $a b$ initio MD simulations typically lead to small model systems and short simulation times. Both give rise to limited configuration sampling which may have severe effects on the statistical significance of the simulation data. Depending on the properties to be explored, the modeler should hence perform a reasonable balance between two diametrically aspects: accurate forces and statistical quality.

Initial criteria to assess the quality of empirical force fields for studying ionic mobility may be the lattice constants and elastic moduli of single crystalline models and the energy required Frenkel for defect incorporation. These properties may be used for comparing force field based simulations with $a b$ initio data. For the sake of computational efficiency, both 
simulations are typically related to zero Kelvin, i.e. the model systems are subjected to simple energy minimization before assessing the propensities described above. More sophisticated benchmarks are given by the evaluation of ionic conductivity and the related activation energy from MD simulation and comparison with impedance spectroscopy experiments. This implies MD trajectories computed at temperature and pressure conditions close to the experiment and reflects a further, highly desired if not indispensable benchmark of the force-fields.

\section{Thermodynamics}

In a crystalline ion conductor, ionic mobility is associated with the spontaneous formation of a Frenkel defect, hence by displacement of an ion to an interstitial position $i$ and formation of a vacancy $v$.

$$
0 \rightarrow v+i
$$

The chemical potential of the Frenkel defects are thus given by the sum of vacancy and interstitial chemical potentials, i.e.

$$
\mu_{\text {Frenkel }}=\mu_{v}+\mu_{i}
$$

To assess chemical potentials from MD simulation data, it is convenient to employ Boltzmann statistics. Differences in free energy (free enthalpy) may be evaluated on the basis of the relative occurrences within a constant temperature (constant temperature, constant pressure) MD simulation run. Thus, by calculating the average density of Frenkel defects and considering the density of the mobile ionic species the difference in chemical potential for reaction (Eq. 2) is given by:

$$
\mu_{\text {Frenkel }}-\mu_{0}=k_{B} T \cdot \ln \left(\frac{\left\langle\rho_{F}\right\rangle_{\mathrm{MD}}}{\rho_{\text {ion }}}\right)
$$

where the average $\left\langle\rho_{F}\right\rangle_{\text {MD }}$ is taken from a sufficiently long MD trajectory to ensure accurate sampling of $\rho_{F}$. 
A simple way to assess the appropriate sampling is given by dividing an MD trajectory in several parts and comparing the corresponding averages separately. This also allows the estimation of error margins for the chemical potential difference as derived from Eq. 4.

In particular for compounds of low ionic conductivity, the required sampling times often turn out to be extremely long compared to the typical ns- $\mu$ s scope of an MD simulation (using force-fields, ab initio MD often reach a few ps, only). Thus, to improve the statistical quality of sampling $\left\langle\rho_{F}\right\rangle_{\mathrm{MD}}$ a common trick is to apply high temperature. In principle the same procedure is applied in the experiments. However, the temperature of the MD simulation runs may be (and often have to be) chosen even larger than experiments would allow. Within the short time scale of an MD simulation, the system may remain in a metastable state and the sampling of solid state ionic mobility may often be performed at temperatures above the actual melting point. Moreover, typical simulation models do not include bond breaking or formation and thus (artificially) prevent decomposition reactions.

\section{Kinetics}

To actually calculate ionic conductivity, it is in principle possible to discriminate interstitial mobility and vacancy mobility. While this requires geometric criteria for identifying the corresponding positions, it is much simpler to directly calculate the overall conductivity from the diffusion constant of all ions.

$$
\sigma=\sigma_{v}+\sigma_{i}=\frac{c_{i} z_{i} \cdot D_{i} \cdot F^{2}}{R \cdot T}+\frac{c_{v} z_{v} \cdot D_{v} \cdot F^{2}}{R \cdot T}=\frac{c_{i o n} z_{i o n} \cdot D_{i o n} \cdot F^{2}}{R \cdot T}
$$

Here $c_{i}, z_{i}, D_{i}$ and $c_{v}, z_{v}, D_{v}$ refer to the concentration, charge and diffusion constant of the interstitials and vacancies, respectively. Depending on the way how the interstitial and vacancy diffusion coefficients are sampled from molecular simulation, it may become necessary to consider a correction factor $k_{c o r r}$ to account for the interactions between the charge carriers [9]. This particularly holds for simulations in which the migration of a single carrier is explored.

$$
\sigma_{v}+\sigma_{i}=k_{c o r r}\left(\frac{c_{i} z_{i} \cdot D_{i} \cdot F^{2}}{R \cdot T}+\frac{c_{\nu} z_{v} \cdot D_{v} \cdot F^{2}}{R \cdot T}\right)
$$


On the other hand, on may directly calculate the average diffusion constant $D_{\text {ion }}$ of all ions of the mobile ionic species. If this is based on sufficiently large simulation models, the interplay between nearby and remote charge carriers may be observed directly (see also section 6 and figs. 1-2). Moreover, no correction terms are required as the effective diffusion constant already accounts for the charge carrier interactions:

$$
\sigma=\frac{c_{i o n} z_{i o n} \cdot D_{i o n} \cdot F^{2}}{R \cdot T}
$$

Comparing the Arrhenius plots of $\sigma \cdot \mathrm{T}, \sigma_{\mathrm{i}} \cdot \mathrm{T}$ and $\sigma_{v} \cdot \mathrm{T}$ yields:

$$
\begin{aligned}
& i: \quad k_{B} T \cdot \ln \left(\cdot \sigma_{i}\right\rangle \mu_{i}+G_{i}^{*} \text {,Diffusion } \\
& v: \quad k_{B} T \cdot \ln \boldsymbol{\Upsilon} \cdot \sigma_{v} \overline{\bar{\gamma}} \mu_{v}+G_{v}^{*, \text { Diffision }} \\
& \text { all ions : } \quad k_{B} T \cdot \ln \left\langle\cdot \sigma \neq G_{\text {all ions }}^{*, \text { iffision }}\right.
\end{aligned}
$$

Thus when considering interstitial or vacancy migration individually, both the migration barrier and the formation energy must be considered. On the other hand, the activation energy of the average diffusion constant of all ions already contains the formation energy of Frenkel defects according to Eq. 3. (Note that $c_{i}$ and $c_{v}$, but not $c_{i o n}$ change exponentially with temperature)

\section{Complex simulation systems}

Single-crystalline models typically require the setup of a supercell model to ensure a reasonably large simulation system. When applying periodic boundary conditions, selfinteraction of the vacancy and interstitial defects must be avoided. This can typically be provided by choosing box dimensions of several $\mathrm{nm}$ or more. Because of ionic migration, the self-interaction of Frenkel defects cannot be avoided. However, sufficiently large model systems may accommodate several Frenkel defects at the same time and thus allow recombination of interstitials and vacancies originating to different Frenkel defects.

Amorphous models may be prepared from a single-crystalline supercell by means of hightemperature MD simulation to induce melting, followed by quenching to reach a glassy state. 
A similar strategy was recently demonstrated for polycrystalline models [5, 6]. To promote the formation of crystalline domains, here quenching is replaced by somewhat slower cooling combined with the application of high-pressure.

Moreover, the preparation of defined nanostructures was recently outlined for $\mathrm{CaF}_{2} / \mathrm{BaF}_{2}$ nanolayer systems [4]. In this study, we first prepared sandwiches of single-crystalline $\mathrm{CaF}_{2}$ and $\mathrm{BaF}_{2}$ layers and then promoted structural relaxation by repeated heating and annealing circles until convergence was reached. The resulting system exhibited a pattern of dislocations to reduce the stress induced by the mismatching lattice constants of the heterosystem.

\section{Mechanistic investigations}

The elaboration of the conductivity mechanisms clearly depends on the model system under investigation. For crystalline compounds, nearest neighborhood analyses offer the possibility to calculate coordination numbers for individual atoms as a function of time. Thus, simple geometric criteria may help to identify interstitials and vacancies. On the other hand, amorphous systems may be characterized by a statistic of ion-ion contacts which may help to discriminate particularly stable constellations from more mobile regions.

Fig. 1 illustrates an nm-sized simulation model (with periodic boundaries) mimicking a $\mathrm{CaF}_{2}$ crystal [10]. The ionic pathways within a time-sketch of $10 \mathrm{ps}$ are illustrated by solid lines. From this the calcium ions may be seen to undergo vibrations only, whilst the fluoride ions display a complex pattern of multiple Frenkel defect formation/recombination and vacancy transport events. The latter is also shown in Fig. 2, highlighting an individual Frenkel defect formation event, followed by subsequent vacancy migration whilst the interstitial is pinned [10].

While ionic conductivity in fluorite is achieved via a single interstitial type, more complex compounds may provide a variety of different interstitial sites. By the example of LiArgyrodites we recently demonstrated the application of Boltzmann statistics to assign free energy levels to different interstitial types [11]. The corresponding energy levels were then associated to NMR and impedance spectroscopy. This allowed to discriminate local mobility 
and real ionic conductivity, i.e. locally confined vibrations and occupation of low energy interstitial sites and ion migration throughout the whole argyrodite unit cell [12].

\section{Conclusions}

While $a b$ initio MD is still far from computationally feasible to provide sufficient statistics, the increasing number of high-quality force-fields allows the study ionic conductivity for a steadily increasing variety of compounds. This is documented by a large number of successful applications of molecular simulation techniques. While detailed references to such studies are beyond the scope of the present concept paper, we outlined the MD simulation methodology and connected approaches to explore ionic mobility. MD simulations offer atomic level of detail insights into ionic mobility and may help to rationalize experimental findings in particular for complex ion conduction mechanisms. The model simulations may be confirmed from thermodynamic and kinetic properties, namely chemical potentials, conductivity and activation energies. Comparison with experimental data is needed, as limited statistics and limited accuracy in evaluating atomic interactions may produce severe artifacts [13].

\section{Acknowledgments}

The author wishes to thank Joachim Maier and Paul Heitjans for stimulating discussions. 


\section{References}

1. Maier J (2005) Nature Materials 4:805-815

2. Guo XX, Matei I, Lee JS, Maier J (2007) Appl Phys Lett 91:103102 (1-3)

3. Voronin BM, Volkov SV (2001) J Phys Chem Solids 62:1349-1358

4. Zahn D, Hochrein O, Guo X, Maier J (2009) J Phys Chem C 113:1315-1319

5. Sayle DC, Doig JA, Parker SC, Watson GW (2003) Chem Commun 15:1804-1806

6. Sayle DC, Doig JA, Parker SC, Watson GW (2005) Phys Chem Chem Phys 7:16-18

7. Haile JM (1992) Molecular dynamics simulation, Wiley

8. Verlet L (1967) Phys Rev 159:98-103

9. Lonercan MC, Shriver DF, Ratner MA (1995) Electrochim Acta 40:2041-2048

10. Zahn D, Hochrein O (2009) Solid State Ionics 180:116-119

11. Kong ST, Deiseroth HJ, Reiner C, Gün Ö, Neumann E, Ritter C, Zahn D (2010) Chem Eur J 16:2198-2206

12. Pecher O, Kong ST, Nickel V, Weichert K, Göbel T, Reiner C, Deiseroth HJ, Maier J, Haarmann F, Zahn D (2010) Chem. Eur J 16:8347-8354

13. Zahn D, Hochrein O, Kawska A, Seifert G, Grin Y, Kniep R, Leoni S (2007) Sci Tech Adv Mater 8:434-441 


\section{Figure captions}

Fig. 1 Visualization of a molecular dynamics simulation mimicking a $\mathrm{CaF}_{2}$ crystal modeled at $1800 \mathrm{~K}$ and ambient pressure. To illustrate ion transport pathways, solid lines mark the ion pathways for a time sketch of $10 \mathrm{ps.} \mathrm{While} \mathrm{the} \mathrm{calcium} \mathrm{ions}$ exhibit vibrations only, i.e. remain at their lattice positions, the fluoride ions display a complex pattern of multiple Frenkel defect formation/recombination and vacancy transport events

Fig. 2 Snapshots showing a small region of the model system illustrated in Fig. 1. The molecular dynamics run reveals Frenkel defect formation (time set to 0 fs) and vacancy migration (320 fs). While Calcium and Fluorite ions are shown in blue and white, respectively, the selected fluoride ions are highlighted by larger spheres and different colors for better visibility of the mobility mechanism 


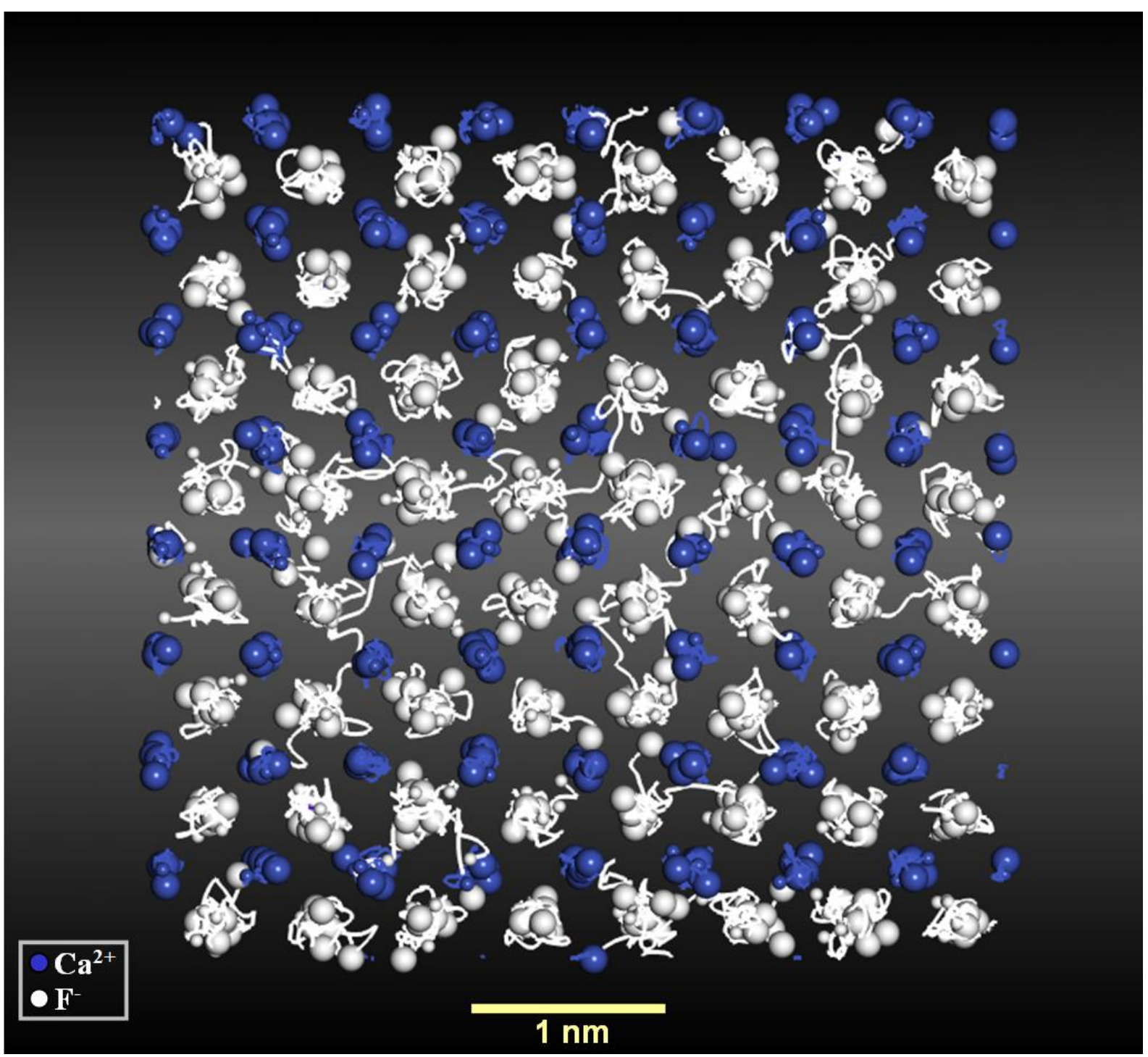




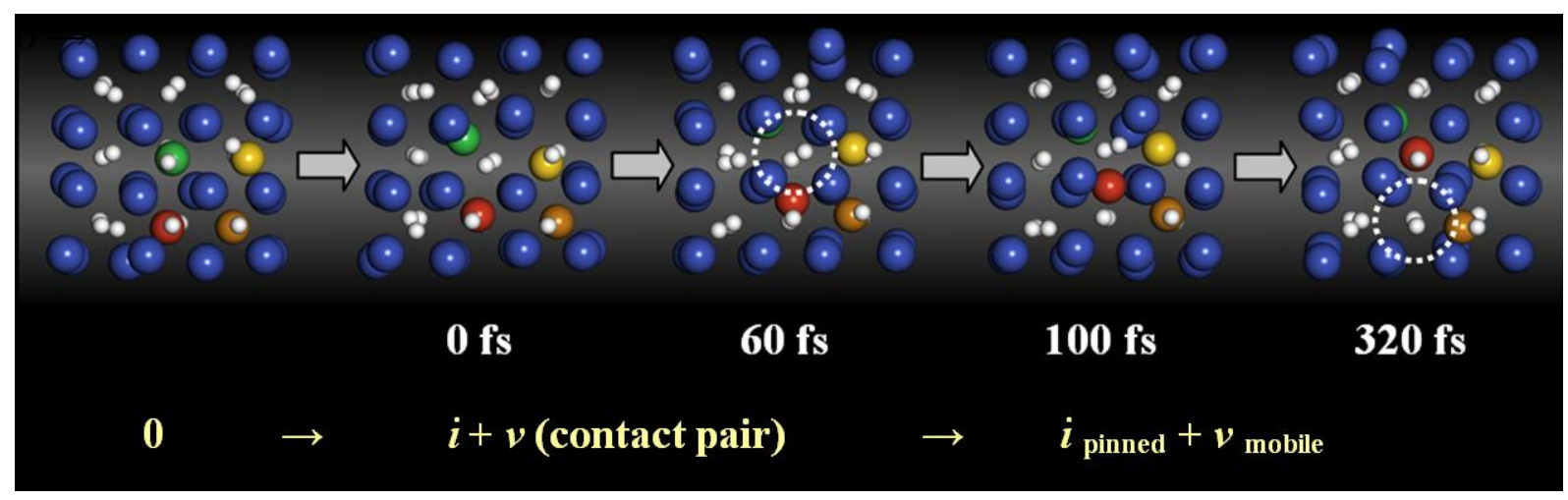

